\title{
Informed consent in genetic research and biobanking in India: some common impediments
}

\author{
PRASANNA KUMAR PATRA \& MARGARET SLEEBOOM-FAULKNER ${ }^{1}$
}

\begin{abstract}
The principle of informed consent, codified in the Declaration of Helsinki, has been widely seen as fundamental to bio-medical and research ethics. The importance of informed consent is increasing in procedures regulating the acquisition, possession and use of personal information, including genetic and medical information. Informed consent, it is believed, ensures that patients and research subjects can decide autonomously whether to permit or refuse actions that affect them. In response to this assurance, there are numerous guidelines at local, national and international levels that recognise the importance of informed consent, especially in research related to healthcare in developing countries. However, complications arise in applying these guidelines to a particular situation, especially under conditions that are prevalent in developing societies, for instance in India. This article discusses common forms of impediments or hindrances encountered while exercising the principles of informed consent in the context of genetic and genomics related research among the tribal and rural caste communities in India. These hindrances include: illiteracy, poverty, paternalistic attitudes, socio-cultural barriers, ineffective regulatory mechanism and procedural inconsistency among others. The data used in this article is based on an ethnographic study conducted between December 2006 and May 2007 using socialscience qualitative research techniques. We observe that three areas require attention: first, the ways in which informed public debate on bioethical issues can be held, and how the application of genetics and genomics in Indian society can be discussed; second, the readiness with which researchers, IRB members and the state appreciate and wish to map the genetic diversity in Indian society; and, third, the risks associated with the application of bioethical principles at a micro-level.
\end{abstract}

\section{Introduction}

Informed consent is an important yet contentious ethical principle in biomedical and genomic research where humans are recruited as research subject participants. This principle is evolving and is discussed and addressed by contemporary international and national bioethical guidelines, taking into consideration the type of populations and technological systems being dealt with. The importance of this principle is to protect the research participants from exploitation, harm and injustice, on the one hand, and to provide a legal and ethical sanction to researchers and institutions to carry out their research and/or business enterprise, on the other. ${ }^{2,3}$ A major concern is whether there can be a true, valid and informed consent procedure that can be followed, especially in the context of illiterate, resource-poor and marginalised peoples and individuals. This article systematically shows a gross mismatch between theory and practical application of the principle of informed consent in settings comprising vulnerable communities in India. Several common hindrances are 
encountered while exercising informed consent in the context of developing countries. ${ }^{4,5}$ This paper has two objectives. It: (a) tries to map the common forms of hindrances encountered while exercising the principles of informed consent in defined social settings in India, and (b) attempts to suggest remedial measures that can strengthen the application of the principle of informed consent in the context of tribal and small scale communities in India.

This paper argues that various stakeholders in the field of bio-medical research in India do not sufficiently appreciate the importance of informed consent and fail to recognise the inherent impediments.

\section{Methodology: target population, period of data collection and analysis}

The data for this article were collected through anthropological ethnographic fieldwork methods and through case studies, open-ended qualitative interviews and focus group discussions with a cross-section of participants that included sample providers, community leaders, researchers and medical doctors in different communities, hospitals, research institutes and universities at various locations in India.

There are around 437 scheduled tribe (ST) communities residing across India. They number around 84.3 million people; $8.2 \%$ of the total population. The literacy rate for ST males is $47.01 \%$ and females $34.76 \%$. ST communities lag behind in most socioeconomic indicators when compared with the mainstream population groups of India. ${ }^{6,7}$ This paper primarily focuses on the applicability of the bioethical principle of informed consent among ST and rural caste communities, presenting the findings of a study of selected communities. This study was conducted in three tribal and two caste communities living in four different states in India: Dhodia tribe of Gujarat; Bhil-Pawara tribe of Maharashtra; Sahu-Teli caste of Chhatishgarh and Kondh tribe; and Agaria caste of Orissa. All the communities selected had participated in some kind of genetic intervention programme, such as a carrier screening programme for sickle cell anaemia, and/or human genome diversity initiatives. Interviews were conducted with researchers and doctors working at well known public and private sector hospitals and research institutes in New Delhi, Hyderabad and Kolkata. Special care was taken in selecting these interviewees who are familiar with or linked to various types of genomics and bio-medical research involving tribal and caste communities in India. No further details on participating communities and individuals can be provided because of identity protection and confidentiality.

This study was conducted between December 2006 and May 2007. Interpreters were used in two community contexts where the participants spoke in their local language/dialect. We do not claim this study to be comprehensively representative of Indian communities or of India in general. However, we do believe that this study represents the general social dynamics experienced by Indian tribal and rural caste communities vis-a-vis bioethical issues in bio-medical research. There were a total of 32 interviews (four university teachers/project investigators, six project directors and 
researchers from various research institutes, 15 community members and seven community leaders); eight case studies and two focus group discussions. Interviewees included university teachers, project investigators, project directors and geneticists. Semi-structured interviews were used to understand the main objectives of the genetic research, procedures followed in subject/ community consultation and challenges faced in data collection. Loosely structured interviews were held with community members and community leaders. The purpose was to understand their motivation to participate in genetic research, the level of information that they received from researchers or data collectors, and their expectations from the research. Case studies were conducted at genomic research institutes, genetic carrier screening centres and in tribal villages. The purpose was to study in detail the interaction between different stakeholders in particular community settings. Two focus group discussions were conducted in tribal and rural caste villages familiar with regular genetic health management programmes, including genetic carrier screening and counselling. There were around 15 participants in each focus group discussion, including people from the community, healthcare institutions, NGOs and genetic health management projects. Broad issues relating to the purposes of genetic research, risks and benefits for the community and issues of informed consent and benefit-sharing were discussed. The collected data was manually coded and analysed with issue-based common themes selected for presentation in this article. Most interviews were audio-recorded and transcribed verbatim and some interviews were transcribed from recollections noted in a field diary. Ethical clearance for this research was received from appropriate authorities before the research was conducted.

\section{Elements of informed consent and related challenges}

The Council of International Organizations of Medical Sciences (CIOMS) guidelines give the most concise definition of informed consent:

\section{A decision to participate in research made by a competent individual who has received the necessary information, has adequately understood the information, and after considering the information, has arrived at a decision without having been subjected to coercion, undue influence, inducement or intimidation. ${ }^{8}$}

The significance of informed consent is based on five basic elements that substantiate its validity in various contexts. The first element is the capacity to consent, where the consent must be given by a person who is legally and factually capable of consenting. The second element is the full disclosure of relevant information, whereby the participant must be informed that the study involves research and of the purpose of that research, the expected duration of participation and the planned follow-up period, foreseeable risks and benefits, and available alternative procedures or courses of treatment that might be advantageous. These must all be explained in the language that the consenting participant best understands. The third element is voluntariness, whereby the participation in research by any individual research participant, group or community must be voluntary and devoid of force and coercion. The fourth element is adequate comprehension of the information by participants, whereby the prospective 
participant must be competent to comprehend the information. The last element is voluntary decision to withdraw from participation at any stage without prejudice to the participant, allowing the subject participants to withdraw from research voluntarily if they feel that their interest is not being respected. ${ }^{9}$

When these elements are tested in practical field situations, one may encounter gross mismatches between ideal elements of the principle of informed consent and practical efforts in exercising this principle. In India, several tribal and caste communities and individuals therein are to the sunjects of genetic intervention as part of genetic screening programmes or genomics-related diversity studies. In most cases, the subject participants are not adequately equipped with information enabling them to consent with competency, nor do the researchers fulfil the requirements in empowering the subject participants by providing adequate and relevant information to enable them to exercise valid consent. This paper tries to identify the common impediments or hindrances that bring about this mismatch, through a thorough and methodological study of views of various stakeholders as cited in the methodology and data collection section of this paper.

\section{Informed consent in an Indian context: some common impediments}

The question of what constitutes a hindrance to genuine informed consent is the subject of heated debate, emanating from situations that give rise to conflict between the interests of the participants and the interests of science and society. ${ }^{10}$ Genuine or valid informed consent is an ideal ethical condition or a moral absolute. A gap between the ideal conditions and a practical situation is what warrants a closer look. This study asks why and under what conditions are the basic elements of informed consent not addressed or fulfilled. Typical hindrances to achieving informed consent include illiteracy, poverty, paternalistic attitudes, cultural barriers, implicit forms of coercion, situational pressure, resistance from healthcare professionals and a lack of or ineffective regulatory mechanisms.

\section{Types of genetic research}

Can informed consent procedures be the same for all types of genetic research? The procedures for data collection, the extent of risk and amount of benefit, and the use, implication and application of genetic information vary for various types of genetic research. Types of genetic research include basic research on the human genome, research into the genetic mechanisms involved in disease, research into the genetic basis of behavioural traits, genetic population research, and trials on genetic therapy. Thus, a particular kind of genetic research may have specific requirements and implications for informed consent. For instance, the relevance and importance of informed consent is different for studies such as genetic epidemiological research among several Indian tribal and caste communities to understand carrier status, genetic diversity and migration history than for research studies and sample collection for genomics and biobanking. Case studies conducted at local levels among participating individuals, community leaders and researchers give us interesting responses. Researchers involved with genetic interventionist studies at community 
level, such as carrier screening for sickle cell anaemia, do not always recognise the importance of informed consent. For them, the collection of blood samples in a screening programme involves minimum risk and is meant for improving understanding the carrier status, educating people about the genetic basis of the disease and for providing pre-marital counselling. As one project director said:

\begin{abstract}
Why do we need to ask for people or their community's consent? We are just helping them through screening programme, we are taking their blood, analyzing it, telling them their disease status and then we are working on the data for better health management. We are not storing the sample or supplying it outside. I do not feel there is any need for informed consent in our case. (Dr D., project director of a sickle cell interventional project in the state of Chhatishgarh)
\end{abstract}

However, researchers involved with sample collection for genomic and pharmacogenomic studies as well as biobanking do recognise the importance of informed consent. They consider that such research involves concern for confidentiality and scope for benefit sharing; hence, a proper informed consent procedure needs to be followed. Therefore, it has become a tricky issue whether to advocate the necessity of informed consent in all types of genetic research or not. For example, as cited in the case above, it is not routine to obtain written informed consent for individuals in a clinical screening programme. However, a particular clinical screening programme that has plans eventually to intervene in healthcare management through pre-marital counselling and pre-natal diagnosis may need to examine further the utility and applicability of informed consent.

\title{
Illiteracy
}

Literacy is universally recognised as a powerful instrument of social change and its level is regarded as one of the most important indicators of social, cultural and health development of a population. Illiteracy can inhibit understanding of research details and methods, undermining the basic requirement of informed consent. In the consentseeking procedure, participating individuals and communities are supposed to be in dialogue with researchers, who try to impart basic knowledge and facts about the study for which subjects are going to be recruited. This dialogue should include discussion about the advantages and the risks involved in the prospective research, and requires a proper understanding and informed decision-making. Illiterate individuals are therefore disadvantaged. Indian tribal population groups, who are more likely to be recruited for genomic research for their 'special' genetic make-up, have a dismal overall literacy rate. ${ }^{11}$ Case studies among certain tribal communities show that their participation in genetic epidemiological and genomic research is often manoeuvred by researchers who do not follow a standard informed consent procedure before recruitment. On many occasions, when asked about the kind of information they have received from research teams to whom they have given their blood or other genetic samples, tribal and illiterate people fail to answer because of lack of comprehension. When asked if they have signed any paper before giving their blood, 
they might reply affirmatively, but fail to explain what the signature was for and what was mentioned on the forms. As one tribal man put it:

Oh! Sir, we are poor, uneducated and unskilful people, what can we understand from that piece of paper? We just followed what the doctor and big people there told us to do. (Mr S.P., 49-year-old male, Dhodia tribe from Valsad, Gujurat, translated by first author)

We recognise that literacy and competency to consent cannot always be equated, and that illiteracy is not necessarily an impediment to understanding or to consent, because it is the responsibility of the investigators (in the case of research) to ensure that the potential participant is made cognisant of the relevant information about the intervention, its risks, benefits etc. However, our field-based observation shows that the condition of illiteracy not only puts people in a disadvantageous position but also makes it challenging for the researchers to avoid acting in a paternalistic way. Additionally, it is questionable whether it is the role of the researchers to explain both the research procedures and the risk implications they might entail for the sample providers.

\section{Poverty}

Poverty is a pervasive problem among tribal and lower-caste people in India. It is not only a question of limited access to the basic amenities of life; it also affects people's ability to make long-term decisions related to health. Poverty undermines the spirit of the original meaning of voluntariness when giving informed consent, if the only way of obtaining medical attention is to take part in genetic screening or participation in any kind of genetic research. In fact, access to healthcare is usually the motive for individuals from marginalised groups to participate in genetic research in India. Case studies among the Sahu caste group from Chhattisgarh state in India show that poor living conditions and poverty induce many to attend health camps at their village and then travel to the district hospital, located some 50 kilometres from their village, to receive some healthcare benefit through a genetic intervention project. They give their blood samples for a carrier detection study, and these can then be used for a molecular level study, without proper informed consent procedures having been followed. When asked why they attended the carrier screening health camp and whether they had signed any consent form, one of them said:

We were told that if we give our blood, we will be given a free medical check-up and free medicine and if somebody is detected as a sickle cell sufferer, that person will receive free medicine for 21 years. We are poor people, so this was great news for us. Hence we decided to participate and donate our blood, otherwise who else will give us such facilities. We were told that this test will help us in knowing if we suffer from the disease or not and we can stop this disease from spreading in our village and community. We did not sign any form and no consent was taken from me or my family members. I do not know if the camp organisers or the doctors have 
talked to any village leader. (Mr G. S., village committee member of a village in Raipur, Chhatishgarh, translated by first author)

Poverty is an important driving force in pushing people to take decisions on their participation in genetic research and on their donation of biological and health information.

\section{Paternalistic attitude}

In India, doctors, scientists and researchers are held in high esteem, especially by rural and tribal people. Their views, suggestions and instructions are taken without question and are respected. In many instances during fieldwork, it became clear that doctors, scientists and researchers have their own way of understanding, interpreting and implementing the informed consent procedures, which differ from the instructions laid down in the guidelines prepared by the Indian Council of Medical Research ${ }^{12}$ and the Department of Biotechnology. ${ }^{13}$ In hospitals, clinics and health camp situations the potential individual participants or donors come into contact with doctors, and they usually leave it up to the doctors to take any decisions on their behalf. They believe it is the doctor who knows better than they when it comes to anything 'medical'. In such a situation it is also hard for the doctor to stick to informed consent procedures. To educate, consult and genuinely inform the individual participants or donors before executing the informed consent procedure puts them in a problematic situation in which they have to take decisions on behalf of the patient and their profession at the same time. Undoubtedly, conflicts of interest arise at times. A medical doctor who was also the project coordinator for a genetic intervention project said:

What's the use of informed consent when many are suffering and dying of diseases? We need to screen them to know their status and take appropriate action for treatment or counselling to ensure that the disease burden is reduced in the society. A mere informed consent will only add to their misery. Do the people who prepared ethical guidelines understand the situation at grass-roots level? (Dr D., project director of a sickle cell interventional project in the state of Chhatishgarh)

The doctor thus regards seeking informed consent as a legal requirement that impedes research and healthcare management, since it requires sufficient resources for informing and educating people about the benefit and risk involved and then winning their trust. He also emphasised that it is 'we', the doctors and researchers, who know better than the subjects what is good or bad in this context.

\section{Cultural barrier}

Culture and social tradition play an important role in the acceptance and feasibility of informed consent procedures at the local level. In certain cultures elderly members of the community, the head of the household or community heads, are supposed to take decisions on matters that in other cultures are treated as personal decisions. Among 
tribal communities, such as Kondh and Bhil, decisions on health and illness are not a personal prerogative, but rather a matter to be decided by the family head or community leaders. Individual decisions bypassing such authorities are considered rebellious. Individuals willing to participate in genetic or genomic research, as donors or research subjects, are expected to have prior approval from the community leadership and once the leaders agree to an action, individuals seldom object to it. Our case studies among tribal communities in India show that genetic research investigation groups always arrange community consultation between the local administration, the tribal leadership and the participating subjects before recruiting subject individuals for study and sample collection. The sampling job of the researcher does not progress without the support of the leadership.

The ideal way to conduct the informed consent procedure is contentious. Some argue for oral or written consent and others favour audio or video recording of the whole procedure. Written consent is the preferred way, but in some societies this is treated with suspicion, because largely illiterate tribal and marginalised communities have experienced exploitation by local landlords and moneylenders, when they had to provide fingerprints without understanding the details of their transaction. As a result, when a genomic research group asks them to sign or print their finger as proof of their consent to participation in a research project as donor, they hesitate.

\section{Ineffective regulatory mechanisms}

Regulatory mechanisms are supposed to safeguard both the interests of researchers, in allowing them to carry out research to further developments in scientific fields, and the ethical rights of the research participants and their communities by protecting them from exploitation and injustice. In India, to carry out biomedical research on human subjects, both the Indian Council of Medical Research (ICMR) and the Department of Biotechnology (DBT), have prepared guidelines to be followed by research projects at clinical and community set-ups. Theoretically, every university, research institute and hospital has its own Institutional Review Board (IRB), which decide whether to approve a research project only after thorough study of the scientific, ethical and legal requirements set out by the two scientific bodies. An IRB is supposed to be independent of the research group that wishes to undertake a research project. The IRB includes members from various cross-sections of academia and society who can take decisions professionally with a concern for social sustainability. Our discussions with IRB members at several universities and research institutes revealed that some members of the IRBs do not fully understand the ethical and social issues concerned in a project and their deliberations and debates are not rigorous. Rather, approval or refusal of a project, on most occasions, is based on favouritism or nepotism. Of course, there is a great deal of variance among institutes and IRBs across regions and the country. If the IRB's decision-making is not rigorous and strictly binding on the project staff, then it gives a free hand to the researcher to manipulate the informed consent procedures at the local level, especially in situations where recruited subject participants are illiterate or drawn from poor backgrounds. At the same time, there is a growing concern among project leaders that if the IRBs are 
given enough authority to sanction research, they may hamper scientific growth, as unprofessional members could block the projects from taking off using ethical and social concerns as a pretext.

Another area of concern is the lack of interim monitoring systems. In India, the culture of internal IRBs is of recent origin, especially at the level of universities and research institutes. Once a project is approved, the IRB has no mechanism in place to monitor the project to evaluate how ethical and social concerns raised are dealt with at the field level.

\section{Individual or/and community}

Who can provide consent is a contentious issue in genetic and genomic research. Researchers are divided on the issue of whether to take informed consent from individuals or communities or both. A consultation document for the UK Department of International Development Commission on Intellectual Property commends procedures for obtaining group consent as well as individual consent, but also remarks that group consent is not a substitute for individual consent. ${ }^{14}$ But there is a growing emphasis on community consent in some situations. Collectivist cultures in parts of Asia and Africa place little value on personal autonomy, so that proxy consent of local authorities, leaders or government officials replaces first person consent of individual community members - although some studies show that collectivist societies do not unconditionally reject individuality. ${ }^{15}$

The requirement for group consent has been recognised by the Tri-Council of Canada, ${ }^{16}$ by Taiwan Bio-bank ${ }^{17}$ and by the Human Genome Diversity Project, ${ }^{18}$ which supports the principle that population consent, as well as individual consent, should be sought for genetic research. ${ }^{19}$ Many argue in favour of community consent, either in place of individual consent or supplementary to it. For instance, Wilkinson said that the way in which one might protect communities is through the consultation between researchers and communities or their leaders, before drafting the project, during the project period and during the preparation of the results. ${ }^{20}$ The challenge is whether a researcher, having consulted with a community, is allowed to ignore its recommendations and proceed with an unmodified proposal. If not, the community has the power to veto it in practice. However, the requirement of community consent usually seems the most restrictive of individual freedom and may violate the rights of an individual who wants to participate. ${ }^{21,22}$

In the Indian situation, the concept of community consent is borrowed from methods of traditional anthropological fieldwork to achieve quick penetration in the community, and to obtain approval smoothly for the recruitment of subject participants. It undermines the value of the individual informed consent procedure and hastens the data gathering process ${ }^{23}$. As one researcher opined:

If we plan to take some sample from a community, we first approach the village or community headman, the local teacher, local representative or key person who can convince their own people, act 
as a buffer if there is any resentment, and give some kind of protection to us and help in easy conduct of research. Once they give the permission, getting individual support becomes very easy. It is crucial in community research. (Mr. B.D., PhD research scholar entrusted to collect blood samples for a genomic research project)

About community consent, a project director of a human genome diversity study said:

In genetic data generation, we just have informal consultation with the village/community leaders such as village headman, local school teacher or local-level Panchayat representatives. Though we give equal importance to individual consent, but, at times with community leaders' approval and consent you can ignore or bypass the individuals. (Dr M., Professor of Anthropology and coordinator of a projects on genomics at an university in Raipur)

Concepts such as community engagement and community consultation are ethical watchwords used in population-based studies of human genetic variation, where the theoretical aim is to allow human populations, those who are the subjects of research, some meaningful control over the initiation and the conduct of the study. The theoretical aim of community engagement or consultation is to enhance the population's control over the ways in which its members are studied. ${ }^{24}$ However, in practice, community consent becomes a strategy for the recruitment of individuals as subjects of genetic research. In the Indian context, any attempt to define a 'community' or a 'group' is problematic, since there are many population categories that overlap in their cultural, social, political, economic and other characteristics. On top of that, it is very difficult to recognise a 'culturally' or 'politically' appropriate authority ${ }^{25}$ having the right to speak for all members of a specified community or group under study.

\section{Procedural inconsistency}

The documentation of informed consent is not a substitute for the detailed procedures of procuring consent. The ethical validity of informed consent hinges not on the written word, but on the quality of the interaction between a patient and a clinician or a subject participant and a researcher, and recordkeeping is just one part of the process. Informed consent is based on mutual trust between the investigators and the participants. In India, we witnessed several lapses in the whole process of the informed consent-seeking procedure, which emphasises documentation rather than seeking qualitative improvement in dialogue and trust between the researcher and the subject. At the community level, it is the researcher who decides which type of procedure best suits his interest, whether oral, written, video or audio documentation. The interests of the participant individual and group are left unprotected. It is obvious that national-level guidelines cannot specify the nature of consent procedures to be applied, as it depends on the specific research for which informed consent is going to be taken. Hence, it remains for the researcher to decide which combination of procedures will best safeguard the concerns of both the subject participants and the researcher. 
Procedures of taking informed consent differ considerably. In some locations oral consent from individuals and/or groups is considered to be sufficient, whereas in other cases oral and written consent are mandatory, and in yet other cases the whole procedure is audio taped or video recorded. During fieldwork in India it was observed that the procedures for informed consent vary among researchers depending on their disciplinary background, institutional affiliation and understanding of ethical principles in biomedical research rather than any standard procedure. Dr M., professor of physical anthropology and coordinator of a project on human genome diversity studies among four tribal communities in central India, said that it is her longstanding rapport with the community that matters most while taking consent. Although she thinks written consent is valuable and desirable, in practice she resorts to community consultation and oral consent. However, Dr P., a prominent figure in genomics research in India and a professor at a leading public research institute in Kolkata, is of a different opinion. He feels that, "since practising written consent is tricky in tribal and rural areas, they cannot but resort to a mixture of oral and written consent processes for which the entire procedure is recorded and video taped". Dr L., director of a well-known public sector genomics research institute who has carried out extensive research on tribal communities in the Andaman and Nicobar Islands of India said, "It is a triangular consultation structure between the community, the local tribal welfare board and our research institute, through which we seek community consultation and generate support for sample collection. Additionally, we also take individual consent."

\section{Blanket consent}

Blanket or 'open' consent is given only once, but covers any use of the material at any time in the future. This is particularly important for scientific research in which new projects or experiments might be devised years after individuals have given their consent and deposited their biological material; they may even have died since then. ${ }^{26}$ The World Health Organization suggested in 1997 that blanket consent might be the most efficient way to allow the use of samples in future projects. However, this suggestion is subject to criticism. One could ask if it constitutes a truly informed consent as legally required in most jurisdictions. Since the potential uses are not known at the time consent is given, can we really consider that the participant received enough information to legitimise any future use ${ }^{27}$

Blanket consent is the most common consent procedure promoted and practised by researchers in India in the field of genomics and DNA banking. Researchers argue that it avoids seeking re-consent in the future and can avoid logistic and administrative problems. Additionally, they feel that true informed consent usually only covers specific and known uses of biological material. Even researchers find it too challenging to keep up with developments in genomics to be able to provide adequate and correct information to subject participants enabling them to give valid consent. But the illiterate and resource-poor participants are not aware of the differences between individual consent and blanket consent and the researchers want to play a safe game. Some contend that blanket consent falls far short of true informed consent, as it is too vague and therefore of little use in legal proceedings. 
Although it may be even further away from the healthcare reality of marginal communities in India, another argument states that blanket consent allow participants to act on their continuing interest in health information. ${ }^{28}$ This viewpoint was expressed by the Quebec Network of Applied Genetic Medicine, which stated that, "Consent is a continuing process and must be reconfirmed, for instance in the cases of significant changes to the research protocol, to the conditions of banking, in the research partnerships, and in the management of the bank." ${ }^{29}$

\section{Retrospective consent}

The issue of retrospective consent is valid in the context of biobanking and, since India is deciding to establish a National Repository (NR) at the central level, this is going to be a serious topic of discussion. ${ }^{30}$ The NR is considering the collection of DNA samples from all anthropologically defined population groups in the country, and these will be stored in a repository. The National Advisory Committee of the NR has already sent its recommendations to the central government for approval. The exact nature and scope of the recommendations cannot be made public before the government's approval. The NR is intended to gather samples, both prospectively and retrospectively, from public, private and individual research centres with a DNA bank. If the plans for the NR are realised, donors and sample providers may need to be asked if they agree to have their samples transferred, in addition to asking their permission for the future use of their samples. Going back to the providers, tracing them and taking re-consent will be a time-consuming, labour-intensive task which is not certain to succeed, and may well turn out to be impractical. In this situation, the NR might resort to the blanket consent, which is the common practice among researchers in India. But, as the new arrangements may have wider ethical implications than those foreseen at the time that blanket consent was given, this may raise issues of confidence concerning the meaning and validity of informed consent.

\section{Discussion and conclusion}

In this paper we have outlined various factors that present challenges to the implementation of informed consent procedures in field situations, especially in the context of tribal and small scale communities in developing countries such as India. Some of the factors are an intrinsic part of the socio-cultural life of the people recruited as research subject participants, and others are extrinsic, coming to the fore as a result of the introduction of new genetic technologies from the outside. These factors are not novel or unfamiliar. It is a matter of identifying and emphasizing them in particular cultural and community contexts for better understanding of the common hindrances in the implementation of consent rather than demanding that procedures fit every context.

It is apparent that the economic, legal, health and socio-political landscape in India provides a different and challenging situation for the application of bioethical procedures, including informed consent. However, there can be no denying that sincere attempts need to be made to make informed consent ethically acceptable in medical and research practice, more so in difficult and diverse cultural, economic and 
legal contexts. It is desirable for the researchers, institutes and institutional review boards involved to understand and appreciate those contexts and decide what kind of model needs to be applied. For example, instead of proposing individual or/and community consent models to a given to a set of research subject participants or their community, it would make sense to take account of the views, aspirations and decisions of those who participate in the whole decision-making procedure. Further, in regard to utility of informed consent in various types of genetic research, we infer that the basic elements of consent are the same in all cases, but in some cases, there may be the need for more extensive provision of information, more time spent explaining the advantages and disadvantages of participating, but never less time and/or information.

In accord with other studies we recognise that the standard accounts of informed consent, standard arguments for requiring consent in clinical and research practice and standard ways of implementing consent requirements can lead to intractable problems, ${ }^{31}$ even more so in the context of communities such as the tribal communities in India that emphasize the importance of familial and cultural groups over individual autonomy. ${ }^{32}$ Informed consent must be grounded in communication that satisfies a wide range of epistemic and ethical norms. ${ }^{33}$ Two areas require immediate further study. One is the creation of an adequate platform to support informed public debate among all the actors on bioethical issues in particular and the application of genetics and genomics in Indian society in general. The second is the readiness of researchers, IRB members and the state to appreciate the enormous diversity that exists in Indian society and to map the different types of hindrances that can emerge while applying bioethical principles at a micro level.

\section{Acknowledgements:}

The authors would like to thank the Netherlands Organisation for Scientific Research (NWO) for providing the funding for the study and all the participants in the field who contributed to this research.

\footnotetext{
${ }^{1}$ International Institute for Asian Studies, Leiden University, The Netherlands; Department of Anthropology, University of Sussex, UK. Correspondence to: pkpatra@rediffmail.com

${ }^{2}$ Hansson, M.G. Building on relationships of trust in biobank research. Journal of Medical Ethics 2005; 31: 415-418.

${ }^{3}$ Andada, P. Module two: informed consent. Developing World Bioethics. 2005; 5(1): 14-29.

${ }^{4}$ Chokshi, D.A. and D.P. Kwiatkowski. Ethical challenges in genomic epidemiology in developing countries. Genomics, Society and Policy. 2005; 1(1): 1-15.

${ }^{5}$ Upvall, M. and S. Hashwani. Negotiating the informed-consent process in developing countries: a comparison of Swaziland and Pakistan. International Nursing Review. 2001; 48: 188-192.

${ }^{6}$ Singh, K.S. 1992. People of India: An introduction. Calcutta, India, Anthropological Survey of India

${ }^{7}$ Census of India. 2001. Office of the Registrar General, India. http://www.censusindia.net (accessed 22 March 2007)
} 
${ }^{8}$ CIOMS (The Council of International Organizations of Medical Sciences). 2002. International ethical guidelines for biomedical research involving human subjects: guidelines 4,5 and 6 .

http://www.cioms.ch/frame_guidelines_nov_2002 (accessed 12 February 2006)

${ }^{9}$ Andada, op.cit. note 3, pp.14-29.

${ }^{10}$ Ibid

${ }^{11}$ Kumar, D. Tribal literacy disparity in India. Current Science 2005; 88: 676.

${ }^{12}$ Indian Council of Medical Research. Ethical guidelines for biomedical research on human subjects. ICMR Bulletin. 2000; 30: 107-116. http://www.icmr.nic.in/guidelines.htm (accessed 14 Sep 2009)

${ }^{13}$ Department of Biotechnology, Government of India. 2002. Ethical policies on human genome, genetic research and services. http://www.dbtindia.nic.in/policy/ polimain.html (accessed $14 \mathrm{Feb}$ 2007)

${ }^{14}$ Dickenson, D. Consent, commodification and benefit sharing in genetic research. Developing World Bioethics, 2004; 4(2): 109-124.

${ }^{15}$ Andada, op.cit. note 3, pp.14-29.

${ }^{16}$ Tri-Council of Canada. 1997. Medical Research Council, Natural Sciences and Engineering Research Council: Social Sciences and Humanities Research Council, Code of Conduct for Research Involving Humans. Ottawa, Canada: Ministry of Supply and Services.

${ }^{17}$ Tsai, D. 2006. Mediating media effects within public participatory opinion in structuring Taiwan Bio-bank. Paper presented at 2006 ELSI Symposium on Re-examining the ELSI Implication of Biobanking - A Cross Culture Perception; held at Taipei 17-18 September.

${ }^{18}$ NARC (North American Regional Committee). Human Genome Diversity Project. Houston Law Review. 1996; 33: 1431-1473.

${ }^{19}$ Godard, B. et al. Data storage and DNA banking for biomedical research: informed consent, confidentiality, equality issues, ownership, return of benefits. A professional perspective. European Journal of Human Genetics. 2003; 11 (Suppl 2):S88-S122.

${ }^{20}$ Wilkinson, T.M. Individualism and ethics of research on humans. HEC Forum 2004; 16 (1): 6-26.

${ }^{21}$ Godard, op.cit, note 20, pp. S88-S122.

${ }^{22}$ Wilkinson, op.cit, note 21, pp.6-26.

${ }^{23}$ Patra, P.K. and Sleeboom-Faulkner M. Genetic biobanking in India - a community based perspective on ways and means of data generation. Taiwan Journal of Law and Technology Policy. 2007; 4: 67-97.

${ }^{24}$ Juengst, E.T. 2003. Community engagement in genetic research: the 'Slow Code' of research ethics? In: Populations and Genetics - Legal and Socio-Ethical Perspectives. B.M. Knoppers, ed. Boston: Martinus Nijhoff: 181-199.

${ }^{25}$ Greely, H.T. Informed consent and other ethical issues in human population genetics. Annual Review of Genetics. 2001; 35: 785-800.

${ }^{26}$ Kegley, op.cit., note 9, pp. 832-836.

${ }^{27}$ Deschenes, M. et.al. Human genetic research, DNA banking and consent: a question of 'form'? Clinical Genetic. 2001; 59 (4): 221-239.

${ }^{28}$ Caulfield, T. Gene banks and blanket consent. Nature Reviews Genetics. 2002; 3: 577.

${ }^{29}$ Cardinal, G. et al. 2003. Statement of principles on ethical conduct of human genetic research involving populations. http://www.rmga.qc.ca (accessed 14 October 2006)

${ }^{30}$ Patra P.K. and Sleeboom-Faulkner M. 2009. The Indian Genomic Biobank Initiative and Emerging Bioethical Issues: a Community-based Perspective. In Human Genetic Biobanks in Asia: Politics of trust and scientific advancement. M. Sleeboom-Faulkner (ed.). London, Routledge: 151-167.

${ }^{31}$ Manson, N.C. and O’Neill, O. 2007. Rethinking informed consent in bioethics. Cambridge, Cambridge University Press.

${ }^{32}$ McGrath, P. and Phillips, E. Western notions of informed consent and indigenous cultures:

Australian findings at the interface. Journal of Bioethical Inquiry. 2008; 5: 21-31.

${ }^{33}$ Manson, op.cit, note 32. 